\title{
Engineering Multifunctional Spacer Fabrics Through Machine Knitting
}

\author{
Lea Albaugh \\ Carnegie Mellon University \\ Human-Computer Interaction Institute \\ lea@cs.cmu.edu \\ Lining Yao \\ Carnegie Mellon University \\ Human-Computer Interaction Institute \\ liningy@cs.cmu.edu
}

\author{
James McCann \\ Carnegie Mellon University \\ Robotics Institute \\ jmccann@cs.cmu.edu \\ Scott Hudson \\ Carnegie Mellon University \\ Human-Computer Interaction Institute \\ scott.hudson@cs.cmu.edu
}
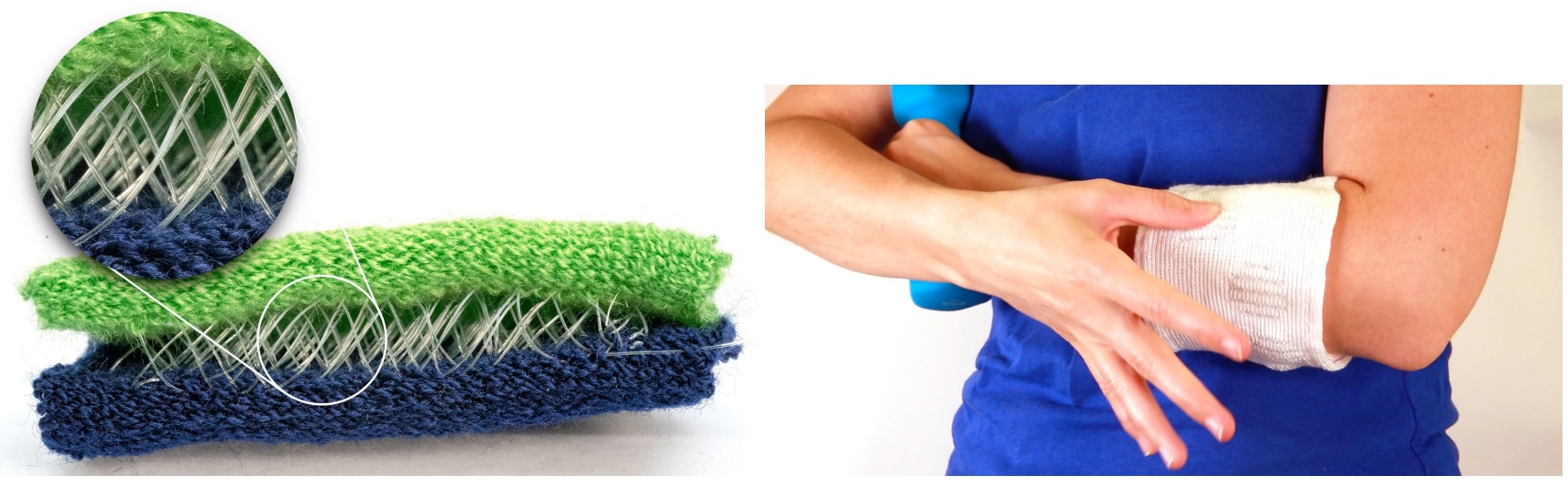

Figure 1: Weft knit spacer fabric (left) can be used as a material in user interface design, by, e.g., by integrating dimensional soft capacitive buttons into a knit surface (right).

\begin{abstract}
Machine knitting is an increasingly accessible fabrication technology for producing custom soft goods. However, recent machine knitting research has focused on knit shaping, or on adapting handknitting patterns. We explore a capability unique to machine knitting: producing multilayer spacer fabrics. These fabrics consist of two face layers connected by a monofilament filler yarn which gives the structure stiffness and volume. We show how to vary knit patterning and yarn parameters in spacer fabrics to produce tactile materials with embedded functionality for forming soft actuated mechanisms and sensors with tunable density, stiffness, material bias, and bristle properties. These soft mechanisms can be rapidly produced on a computationally-controlled v-bed knitting machine and integrated directly into soft objects.
\end{abstract}

This work is licensed under a Creative Common

Attribution-NonCommercial-NoDerivs International 4.0 License.

CHI '21, May 8-13, 2021, Yokohama, Japan

(c) 2021 Copyright held by the owner/author(s).

ACM ISBN 978-1-4503-8096-6/21/05.

https://doi.org/10.1145/3411764.3445564

\section{CCS CONCEPTS}

- Human-centered computing $\rightarrow$ Human computer interaction (HCI).

\section{KEYWORDS}

Textiles; Machine Knitting; Soft Sensors; Knitted Mechanisms.

ACM Reference Format:

Lea Albaugh, James McCann, Lining Yao, and Scott Hudson. 2021. Engineering Multifunctional Spacer Fabrics Through Machine Knitting. In CHI Conference on Human Factors in Computing Systems (CHI '21), May 8-13, 2021, Yokohama, Japan. ACM, New York, NY, USA, 12 pages. https: //doi.org/10.1145/3411764.3445564

\section{INTRODUCTION}

In recent years, computer-controlled v-bed weft knitting has been explored as a powerful method to create highly-customizable textile output $[23,32,37,38]$. These machines require very little per-item setup, allowing one-off production, and are flexible enough to incorporate a wide variety of materials.

Many complex seamless objects can be fully automatically machineknit, and knit fabric can be post-processed with standard textiles manipulations including sewing, cutting, gluing, and heat-setting. The stitch-level controllabity of knitting allows us to consider fabric 


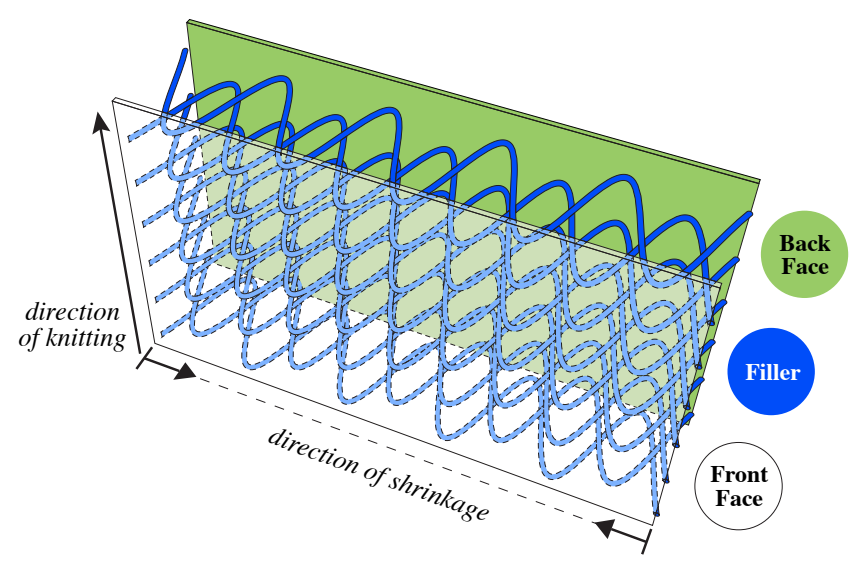

Figure 2: The basic structure of a spacer fabric. Faces are shown in green and white, and filler in dark blue. A face may be removed to produce the "bristles" structure.

as a tunable material: a knitted structure can incorporate selective opacity or elasticity [15], or knitted-in routed tendons [2].

Spacer fabrics, Figure 1 (left), are a category of knit structures that have a unique springy feel, breathability, and low density, making them useful for a range of applications such as uppers of running shoes and padding for orthotics. They are also used as replacement for foam rubbers for their relative sustainability and resistance to degrading [34] and as a structured fiber reinforcement for concrete [53]. However, these fabrics are typically produced on warp knitting machines, which are characterized by dedicated yarn feeders for each needle in a bed; these machines are very large, have relatively high setup overhead, and are typically used to produce undifferentiated yardage with very little within-fabric programmability. By using weft knitting instead of warp, we can tune the production parameters of the fabric directly on a stitchby-stitch basis, incorporating multiple functional characteristics in a given object.

We contribute an explanation of the weft knit spacer structure, an exploration of knitting parameters for tunable mechanical performance, and examples of objects which vary these parameters for mechanical and interaction applications. This work contributes to our understanding of weft knitting as a general-purpose fabrication technology for programmable soft structures with applications ranging from robotics to wearable and architectural functional fabric.

\section{THE SPACER STRUCTURE}

A knit spacer fabric consists of knit faces and a lofty filler, Figure 2. In a classic spacer fabric such as the warp-knit ones in the related industrial work, this filler yarn is semi-stiff and holds the two faces apart at a distance. In this work, we additionally investigate two variants: 1) a single-face variant, the "bristles" structure which we show in sections 7.2 and 7.3, and 2) a variant with soft filler yarn which does not exhibit the lofty thickness, but which can be used as a soft padding or in velcro-like applications as in section 7.3.

\section{RELATED WORK}

\subsection{Bulk Spacer Fabrics in Industry}

As stretchable, compressible, and resilient materials [63] with excellent air and moisture permeability $[30,61]$, spacer fabrics have been used to replace conventional foam rubber in the context of cushioning and body support $[1,54]$ and in architectural acoustics [29].

The main industrial approach to produce spacer fabrics is in bulk using warp knitting [54]; as such, most material characterizations of spacer fabric focus on warp-knit materials (with some notable exceptions - e.g., our findings in section 6.1 improve upon brief claims in [6]). This programmability has benefits for interaction such as integrated electrical routing, and we focus on it as the basis for tunable mechanical properties.

\subsection{Metamaterials}

Spacer fabric (and, indeed, most fabric) is an example of a metamaterial: an aggregate material where designed microstructure influences overall properties. Metamaterials have been an active topic of research in optics [62], acoustics [33], antenna design [12], and mechanics [56]. One popular style of metamaterial fabrication uses repeated, tunable cells. The parameters of these cells can be optimized (e.g., for weight/strength [3] or flexibility [45]); or used to create mechanisms with appropriate design tools [19-22].

One particularly relevant metamaterial structure is "bristles": hair-like structures that have a high aspect ratio suited to transmit and amplify vibrations [41] or as tactile or high-friction surfaces $[26,42]$. We explore bristles as a variant of spacer fabrics in which one face layer is unravelled after knitting, and we look into both velcro-like applications and directionally biased actuations. Other structures that can achieve a similarly biased surface friction and have been used in soft actuations include stretched kirigami patterns [51] and angled origami folds [59].

\subsection{Functional Structures in Textiles}

Within textiles, functional material characteristics can be produced in a number of ways. For example, quilting is a process which consists of layering padding between cloth and sewing through the layers to attach them; the density of stitching has been shown to control the thickness of the resulting structure [11]. In industry, $3 \mathrm{D}$ weaving - a generalization of double-cloth weaving - is used to produce composite reinforcement [7, 27]; recently, researchers and designers demonstrated that these tools can be extended to make 3D-woven shoes [14] with varying functional zones. Further, yarn can be felted [18], fabric sheets laminated [46], or thermoplastic electro-spun and folded [52] into objects with embedded functionality.

Weft knitting, the fabrication technology used in this paper to create spacer fabrics, is remarkably flexible. Knitting machines can create a wide range of 3D topologies, either through careful handdesign [58]; with the aid of primitive-based design systems [23, 32]; or by directly converting 3D models [37, 38, 48]. At the stitch level, knit fabrics can integrate a wide range of surface textures with different mechanical properties such as auxetic behavior [8], which can in turn augment the functionality of designed objects [15]. 


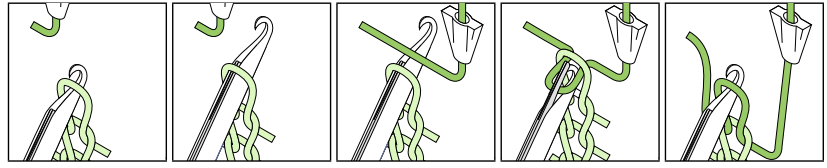

(a) The knit operation

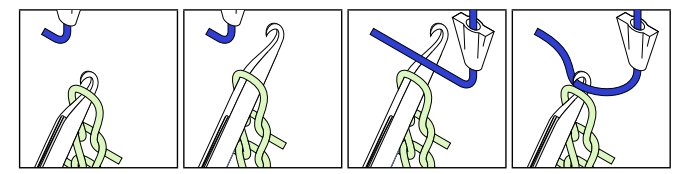

(b) The tuck operation.

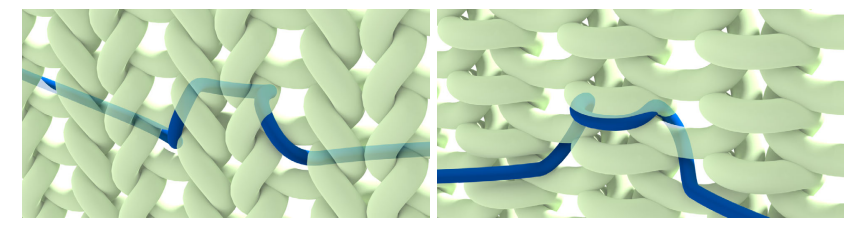

(c) A blue yarn tucked into a pale green knit stitch, shown from the front (outside) and back (inside).

Figure 3: The knit and tuck operations. (a) The fabric faces are formed using the knit operation, which pulls a loop of yarn through a previous loop[s] of yarn (used with permission from [2]). (b-c) The filler yarn is attached with the tuck operation, which does not form a new row of face fabric.

This work focuses on characterizing the specific structure of spacer fabrics.

\subsection{Soft and Deformable Interfaces}

Soft interfaces have been developed for locomotion [36], manipulation [35], display [64] in contexts including prototyping [59], biomonitoring [66] and for generating different physical affordances [9]; the deformability of soft objects particularly supports haptic modalities in contexts such as wearables [5] and mobile devices [9].

While many soft interfaces are elastomeric and are fabricated through casting [64] and layer-based approaches [44], a growing body of work has explored interface fabrication methods based on textiles techniques. For example, interactive fabrics can be embroidered [50], dyed [17], or woven [55]; textiles can incorporate resistive sensing for stretching, bending, and position [4,65], and capacitive sensing of objects $[10,28]$ and gestures $[39,40]$.

Weft knitting in particular can be used with conductive yarns to create sensors [25, 43, 47] and, with integrated tendons, to create actuated structures [2]. Weft knit skin has also been used over foam to create a flexible manipulator for soft robotic applications [24].

\section{PRODUCING SPACER FABRICS ON A V-BED WEFT KNITTING MACHINE}

A v-bed weft knitting machine produces knit fabric on two straight rows (beds) of hook-shaped needles [2,32]. Spacer fabric knitting proceeds by alternating between two basic steps:
- A number of face rows are formed using sequences of the knit operation, Figure 3(a). Rows are added to both the front and back faces, which are knit on the front and back beds respectively. At this time, therefore, the two faces of fabric are separated by only the small gap between the two needle beds.

- Filler rows are added at the same needles using the tuck operation, which incorporates the yarn into the stitch without forming another row of fabric, Figure 3(b). By tucking at a regular interval onto alternating beds, the filler yarn forms a shallow lengthwise zig-zag, Figure 3(c). Because tucking does not add height to the fabric, subsequent passes of filler yarn add density to the same face row of the fabric, as shown in Figure 5.

The faces are knit with an elastic yarn which is in tension at knitting time. After knitting, the tensioned elastic yarn in the faces causes them to shrink laterally as indicated in Figure 2. This shrinkage pulls the filler yarn zig-zag in, pushing the faces apart into the characteristic "fluffy" thickness of the spacer fabric.

\section{FABRICATION PARAMETERS}

As composite materials, spacer fabrics have properties that arise out of the interplay between individual input materials and how they are arranged. As shown in Figure 4, we organize these into material parameters, determined by the yarns used, and geometric parameters, which are controlled by knitting process and therefore ulimately by the machine code.

\subsection{Material Parameters}

The material parameters include the stiffness, friction, and stress/strain properties of the yarns in the fabric. A spacer fabric has at least three component yarns:

- Main Face Yarn[s]: The yarn which forms the faces of the spacer structure can be any machine-knittable yarn. It is trivial to knit the two faces in two different yarns, and possible to mix yarns within a face. The thickness of these yarns will directly contribute to the stiffness of the faces at a given stitch size. These yarns may have additional material properties such as conductivity (for knitted circuits and sensors) and higher or lower friction.

- Elastic: An elastic yarn is required to provide the lateral drawin which produces the lofty spacer structure. The face yarn can itself be elastic, or an elastic yarn can be used alongside a non-elastic face yarn using plating, a way of knitting two yarns into the same stitches [2]. It is possible to tune the tension under which this elastic is knit - elastic knit under tighter tension will draw in more - but the elastic must be able to withstand the strain applied.

- Filler Yarn: The filler yarn forms the zig-zag path between the faces. In order to achieve the lofty spacer form, the filler yarn must be stiff enough to push the faces apart; because it is only tucked into the face loops (not pulled through into new knit loops), it can be stiffer than the face yarns.

In addition to individual yarn properties, the friction between the face yarns and the filler yarn affects the tactile qualities of the spacer fabric. "Stickier" face yarns do not spring back quite as easily, and 


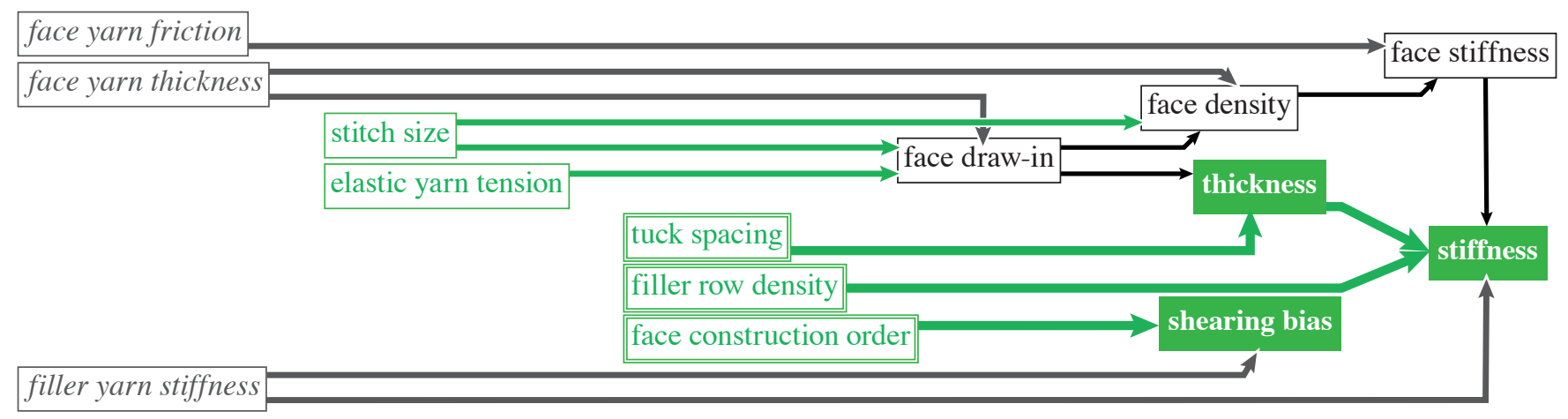

Figure 4: While the structural properties of a spacer fabric are influenced by many factors, we identify several material parameters (shown in dark grey italic text) and knit programming parameters (shown in green text) that can ultimately affect useful fabric characteristics (shown in white bold text).

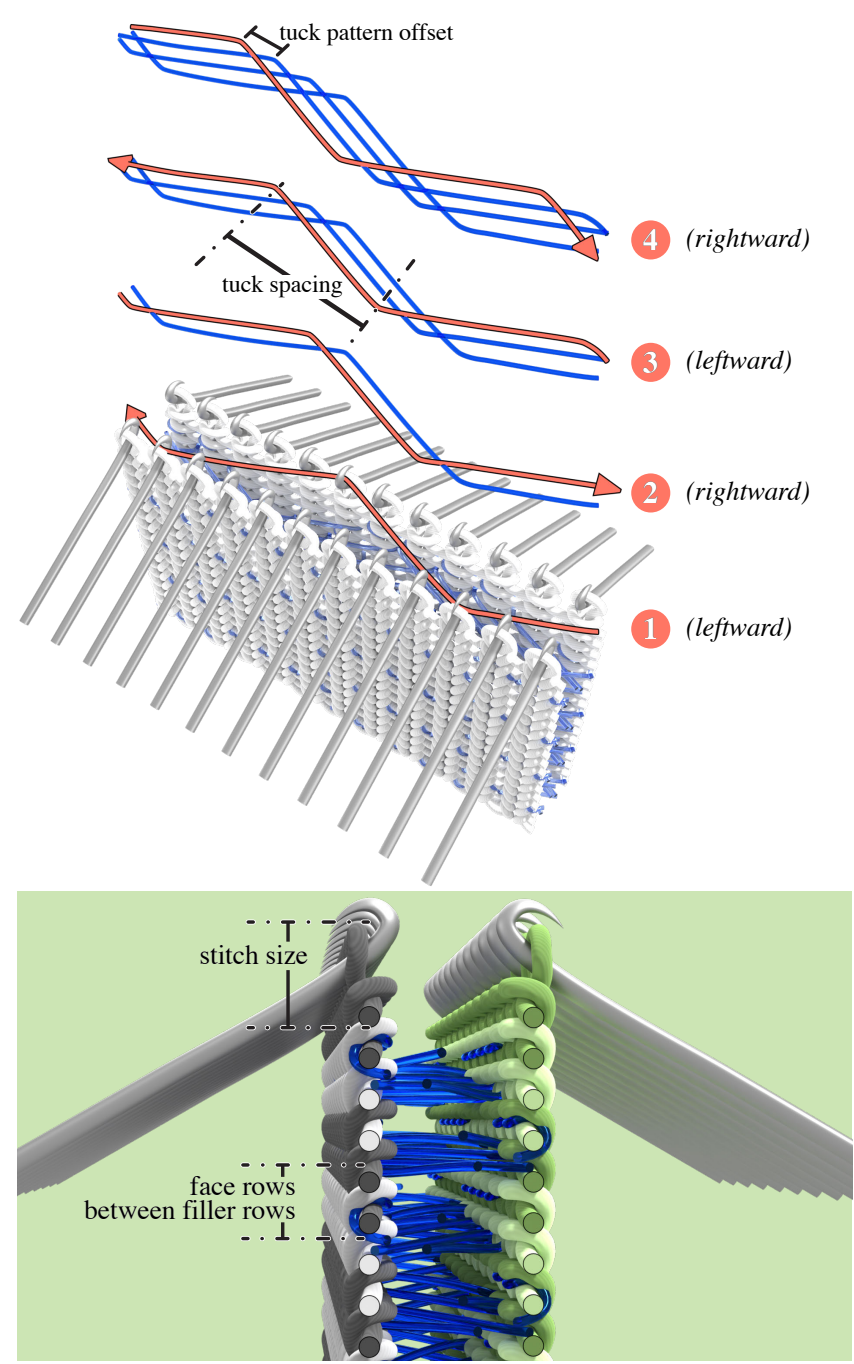

Figure 5: Geometric parameters of spacer fabrics. can be stretched out of shearing bias. For example, we found that the combination of Bekaert VN35X4 conductive polyester yarn and nylon monofilament that we used in the capacitive sensor shown in Figure 14(a) was somewhat "sticky" in the spacer configuration. This gave the button a more malleable feel, with delayed return, than swatches knit with our default acrylic yarn.

As a practical matter, the yarns used for the spacer fabric must be compatible with machine knitting - that is, they must not be too thick for the gauge of the machine, nor too weak or brittle to survive the knitting process. (We list some additional machinespecific considerations in the Appendix [A].)

All examples in this paper were produced on a Shima Seiki SWG091N2 15 gauge industrial knitting machine at half gauge (using every other needle as described in [32]). Unless otherwise noted, we used Tamm "Petit" acrylic yarn with hair-thin $(0.06 \mathrm{~mm} \varnothing)$ latex elastic for faces and KastKing 6lb test monofilament $(0.22$ $\mathrm{mm} \varnothing$ ) for color-tinted filler and Hi-Seas "Grand Slam Mono" 6lb test monofilament $(0.25 \mathrm{~mm} \varnothing)$ for clear filler.

\subsection{Geometric (Programmable) Parameters}

The geometric parameters of spacer fabric, Figure 5, can be altered through different machine code.

- Tuck spacing: The distance in needles between a filler yarn tuck and the next tuck (on the opposite bed). The distance between tucks must be less than six and greater than one to ensure clean tuck formation on our $15 \mathrm{~g}$ machine.

- Filler row density: The ratio of filler rows to rows of face fabric height. As shown in Figure 5, filler rows do not add height.

In knitting, every row has a direction of formation (leftward or rightward) which must be alternated. To greatly simplify the programming of these structures, we always pair a leftward pass with a rightward pass; therefore, in all our examples, the number of rows of each are always even; we factor out this duplication in expressing the filler row density as a ratio. The fabric shown in 5 has a filler row density of 2/1: four filler row passes for every two face passes (leftward and rightward). 
- Tuck pattern offset: The distance in needles between the tuck positions in one pass and the tuck positions in the previous pass.

- Face fabric knitting order: Which face (front or back) is knit first after a set of filler rows.

- Yarn tension: Depending on the knitting machine, it may be possible to control the tension on the yarns during knitting Our Shima Seiki machine allows row-by-row tension settings for the elastic yarn feeder only. Elastic yarns are typically knit under tension; in the context of spacer fabrics, the force induced by this tension causes the face fabric draw-in. We chose a hair-thin elastic for this work to best support mixing with a wide range of other face yarns. We found that our has a narrow range of working tensions without breaking, so in practice, we chose to keep the elastic yarn tension constant. For the non-elastic yarns, tension is typically set to minimize breakage and dropped stitches and it is not altered during knitting.

- Stitch size: The size of knit stitches produced by a knitting machine arises from a combination of factors, including yarn tension and post-processing. The most dynamically adjustable factor is the nominal stitch size, which is the amount of yarn pulled into a loop as it is formed, as determined by programmable stitch cam settings. For a given yarn thickness and friction, stitch size affects the density and stiffness of a knit fabric. Within a spacer fabric, face fabric density can affect draw-in, as a fabric that is already quite dense may not be able to draw in further.

While a given knitting machine has inalterable physical spacing between the needles, the stitches can be made farther apart by integral multiples of this spacing; in this work, we knit at "half gauge," with intervals of one needle between stitches. We do this both to allow for ease of shaping operations (as described in [32]) and to promote good draw-in.

All examples in this paper were produced using Knitout control instructions [31] generated with JavaScript code. To keep our explorations tractable, we decided to maintain constant yarn tensions, tuck pattern offset (1), nominal stitch size (40, with leading set 25), and half gauge throughout the presented work.

\section{RESULTING SPACER FABRIC CHARACTERISTICS}

As described in the previous section, some of these parameters must be kept in a narrow range to ensure knittability (e.g., elastic yarn tension), and we kept some constant in order to keep our explorations tractable (e.g., stitch size). However, others can be altered to affect the overall characteristics of the composite spacer fabric structure. In the following sections, we outline such characteristics and describe how underlying production parameters can affect them. These effects are particularly useful in the case of the geometric/programmable parameters: because these can be altered with machine instructions, hence they can be varied within a given knit object. We provide an overview of the relations between knitting parameters and resulting fabric characteristics in Figure 4.

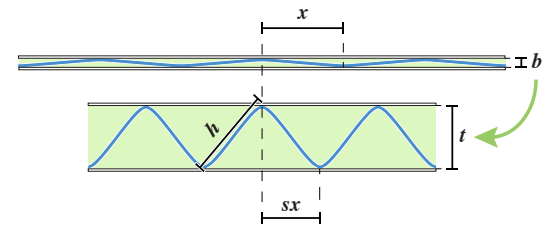

(a)

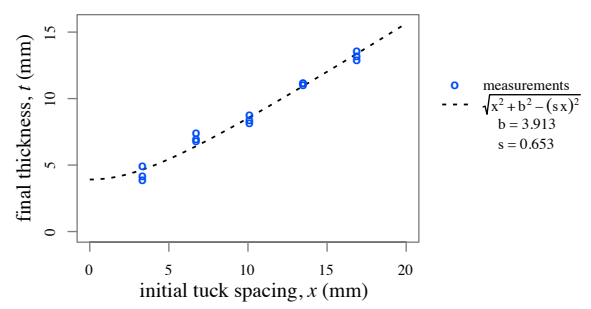

(b)

Figure 6: (a) The spacer fabric "draw-in transition" can be modeled by thinking of the filler yarn as the fixed-length hypotenuse of a right triangle whose base shrinks as the face fabric shrinks. (b) This simple model fits measured test data.

\subsection{Thickness}

A defining characteristic of spacer fabrics is their thickness. While the thickness of the individual faces can be altered with thicker or thinner face yarns, the dominant factor of the overall spacer fabric thickness is the distance between the faces.

Assuming a filler yarn that is semi-stiff, such as a nylon monofilament, this distance is determined by two factors: the spacing of the tucks, and the lateral draw-in of the face fabrics induced by their elastic. As illustrated in Figure 6(a), when viewed from the top, the filler yarn layout can be diagrammed as triangular struts. We consider each section of filler yarn as the hypotenuse of a right triangle. When the fabric is knit, the tuck spacing sets the base length $x$ of this triangle, and the gap between knitting machine beds sets its height $b$ ("bed gap"). As the hypotenuse, the filler yarn has length $h=\sqrt{x^{2}+b^{2}}$. When the face fabric is drawn in by factor $s$ to final length $x^{\prime}=s x$, the filler yarn remains the same length, resulting in an overall fabric thickness of

$$
t=\sqrt{h^{2}-x^{\prime 2}}=\sqrt{x^{2}+b^{2}-(s x)^{2}}
$$

The lateral draw-in of the fabric is determined primarily by the tension of the elastic yarn at knit time and to a lesser extent by the face yarn thickness and stitch size. As described in section 5.2, the range of possible elastic tensions for a given elastic may be narrow. The spacing between tucks also has practical limits (too small and it's not a spacer; too large and the tucks may not form cleanly) but there is variability possible within that range.

In order to test this model, we created sample swatches with tucks spaced 1,2, 3, 4, and 5 stitches apart, with other design factors held constant, and measured the thickness of each sample in three locations. Our proposed model is a good fit to these thickness measurements (Figure 6(b)); and, further, the coefficients of the model (bed gap $b=3.913 \mathrm{~mm}$, shrink factor $s=0.653$ ) are close 


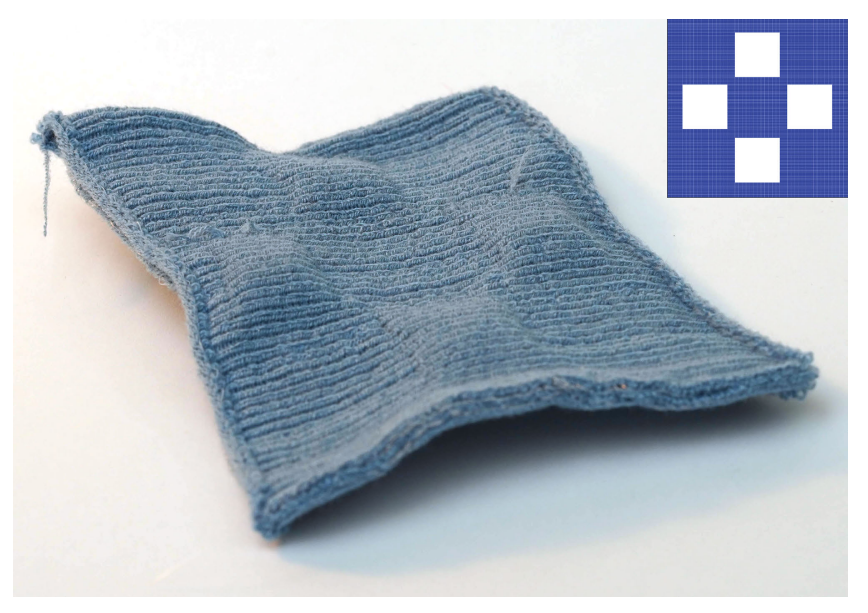

Figure 7: Differences in tuck spacing can be used to generate fabrics with areas of different thickness.

to the measured values for our machine (bed gap of $\approx 4 \mathrm{~mm}$ ) and test fabric (shrink of 68\%); suggesting that the model might work equally well as a predictive model given just this information.

Because thickness can be varied with tuck distance changes, different thicknesses can be implemented within a fabric without special attention to yarn carriers or extra yarn-inserting maneuvers. This allows thickness-based surface patterning such as the example in Figure 7.

\subsection{Stiffness}

Spacer fabrics are also notably stiffer than non-spacer fabrics knit with comparable yarns. Stiffness is the result of several properties: the stiffness and stretchiness of the face yarns (determined by face yarn, stitch size, and tension), the filler row density, and the thickness of the resulting structure (determined by tuck spacing, as described above).

To test these proposed stiffness parameters, we knitted swatches varying tuck spacing and the ratio between face rows and filler rows. Because we found that fabrics with multiple monofilament tucks on a needle at the same time consistently had problems knitting cleanly, we excluded cases in which close tuck spacing combined with higher numbers of filler rows resulted in re-visiting a tuck location before knitting face fabric at that location.

We additionally tested swatches with 1a) filler yarn tucks all on the same bed and $1 \mathrm{~b}$ ) no filler yarn at all, to isolate the effect of the monofilament's stiffness unaffected by the spacer thickness; and 2) no elastic yarn at all, to isolate the effects of face fabric density and elastic recovery. These were all knit with the same number of stitches per face and the same stitch size; the differences in overall swatch dimensions show the extent of elastic draw-in relative to the elasticless swatch.

We tested each swatch for bending stiffness in both horizontal and vertical directions. Each swatch was positioned in a highfriction (sandpaper) rig and clamped with a light (150g) weight, Figure 9. Successive force was applied until the bottom edge of the swatch was level with a $45^{\circ}$ line from the clamped edge. We chart results in Figure 8. Overall, both thickness and row density indeed

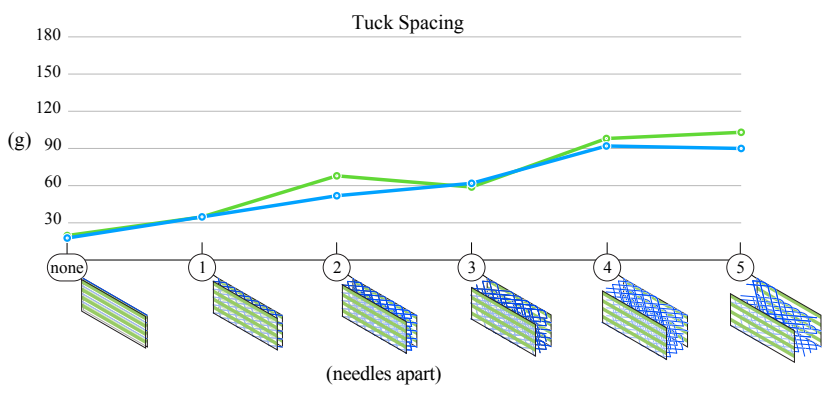

(a)

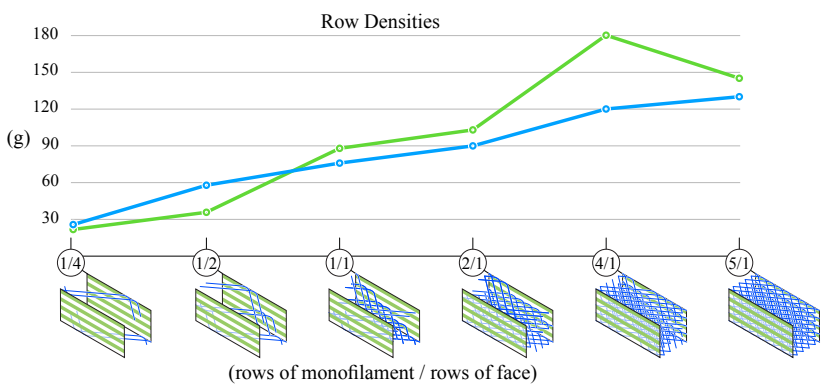

(b)

Figure 8: Mass required to bend stiffness samples to $45^{\circ}$ in the bending rig. Blue lines chart bending in the horizontal direction (along a row); green lines chart bending in the vertical direction (along a column of stitches). (a) Varied tuck spacing at a constant 2/1 row density; (b) varied row densities at a constant five needle tuck spacing.

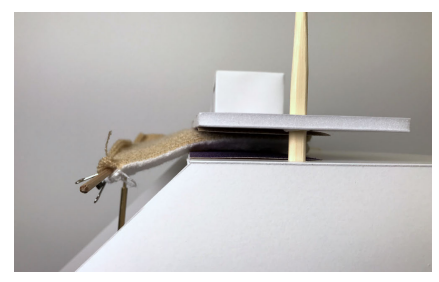

Figure 9: For stiffness testing, each swatch was lightly clamped to its center line between two sandpaper surfaces. The free edge was loaded with successive masses until it bent below a $45^{\circ}$ reference line.

affect stiffness. Because comparable stiffnesses might be achieved with either row density or tuck distance manipulations (compare the swatch with $1 / 4$ row density at tuck spacing 5 to the swatch at 2/1 density with tuck spacing 2) spacer fabrics offer some aesthetic freedom in how stiffness differentials might be arranged.

\subsection{Shearing Bias}

Spacer fabrics can have a slight but noticeable mechanical bias in the vertical direction: under pressure, the fabric will tend to shear to the same direction. 


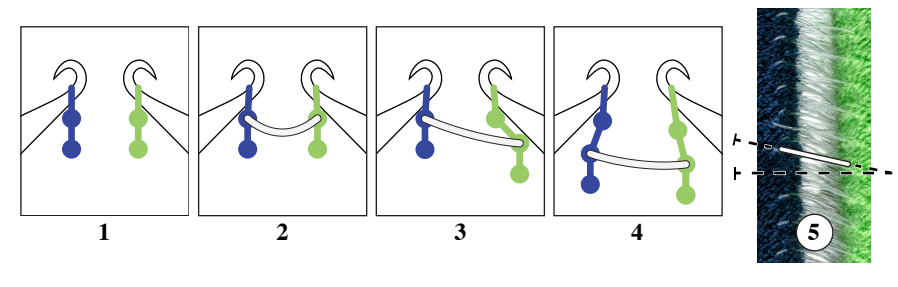

Figure 10: Bias formation in spacer fabrics. 1: The knitting machine beds shown from the side, with two rows of face fabric on each bed. 2: A row of filler yarn has been added; the springy filler yarn attempts to push the cloth apart, but cannot (owing to the close spacing of the needles). 3: Adding the next row of one face (shown in green) allows the filler yarn to spring outward, skewing the connection in one direction. 4: When the next row is added to the other face, the skew remains and reinforces the skew of future rows. 5: This skew bias is visible in the final fabric, shown from the side. In this swatch, the right (green) face will shear downward.

This bias can be produced by altering the order in which the face fabrics are formed near the filler row, Figure 10. The face which is knit first will tend to shear downward. The bias can be minimized by alternating between face-knitting order, and the opposite bias can be introduced after a gap of face rows without filler rows joining them.

This effect is reasonably repeatable with the acrylic yarns we used, though we have two important notes for other practitioners: (1) having unequal numbers of front and back tucks in the first spacer course (which can happen for spacer regions of certain widths) seems to override the effect of face order entirely; (2) occasionally (10-20\% of the time), the shear bias of a spacer patch will be flipped from what we would predict otherwise; re-knitting the same pattern will often produce the expected bias. (One can also clamp a sample in the desired orientation to re-set its bias; though no examples shown in this paper have been clamped.)

\section{APPLICATIONS}

In addition to the programmable characteristics described above, yarn properties and post-processing can widen the range of applications for spacer fabrics.

\subsection{Soft Linkages}

The bias in spacer fabrics produces a directional shearing motion under pressure. We demonstrate this effect in Figure 11: sections of different bias can be arranged, and the fabric folded, to produce a mechanism based on shearing linkages. In one folding configuration, squeezing the fabric tends to shear the outer layer away from the inner, causing the overall fabric to bend.

\subsection{Robotic Skins}

Our "bristle" structure is formed the same way as the spacer fabric, but one face is unraveled after knitting. The resulting structure is very similar to a "plush" or "terry" knit, but with much longer loops than are typically produced in these processes. Knitting a sacrificial face helps ensure that the bristles are formed properly; without it,
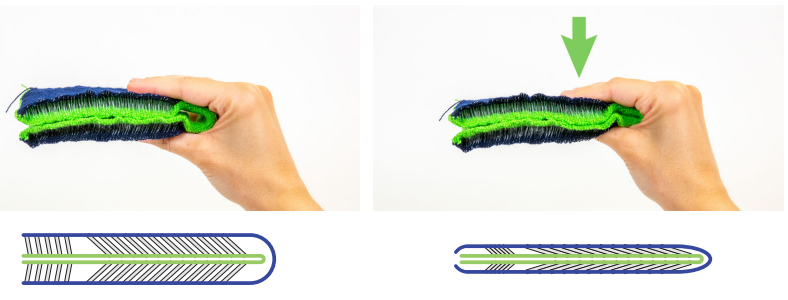

(a)

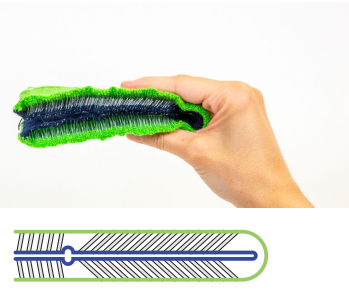

(b)

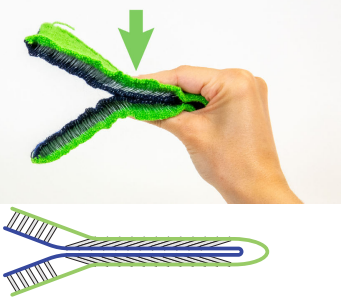

Figure 11: Bias in spacer fabrics can be used to make anisotropic shearing linkages.

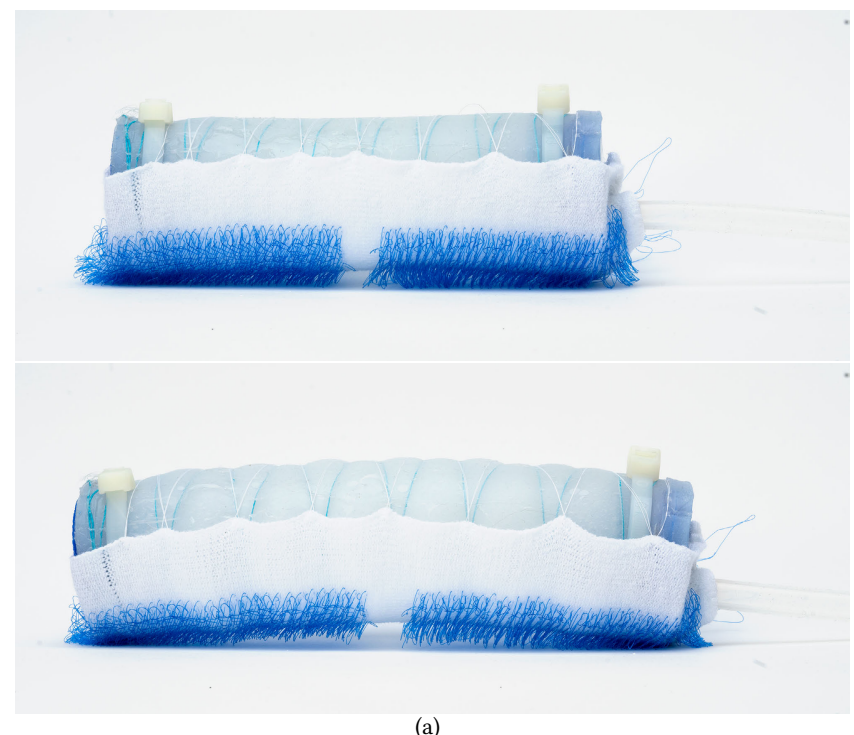

(a)

Figure 12: Biased bristles as cladding on a pneumatic extension actuator.

the monofilament may not drop cleanly off the needles after each row. The most predictable results are achieved if the "sacrificial" unraveled face was knit in the same kind of yarn as the remaining one.

Inspired by kirigami-clad pneumatic mechanisms, [51], we applied a biased bristle skin to a fiber-reinforced elastomeric extension actuator [16] Figure 12(a). When the actuator is pressurized, it stretches uniformly; when it is allowed to relax, the bristle structure acts as a ratchet and keeps the front part of the actuator in place 


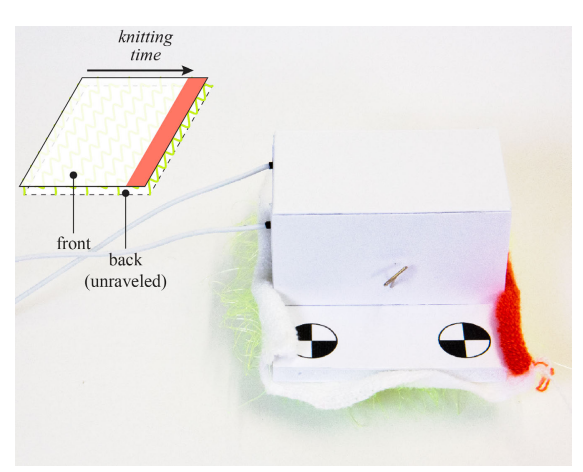

(a)

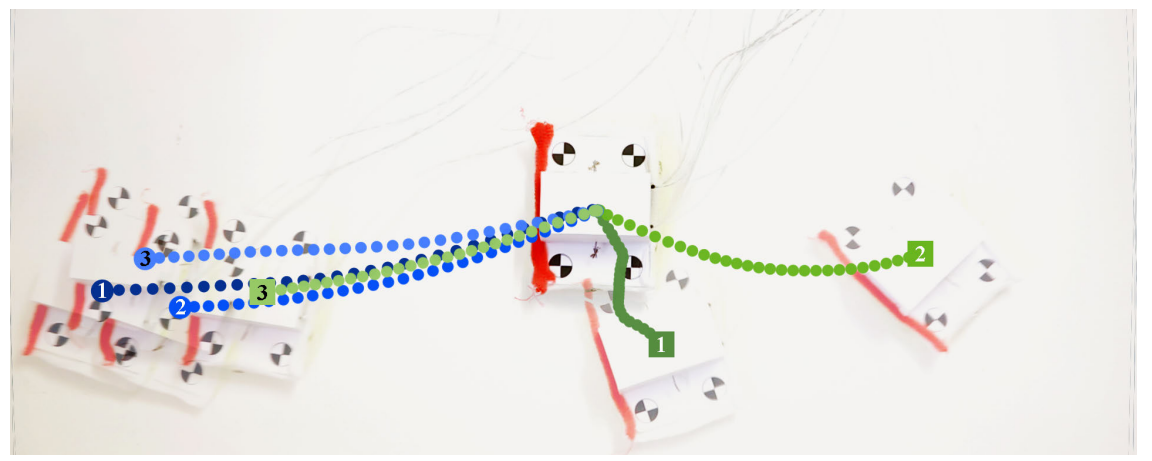

(b)

Figure 13: Varying the face knitting order and bristle height can produce bristle-bots with different travel directions. Paths are plotted over the first 1 second of travel. Dots are placed every 30th of a second. Blue paths marked with a circle indicate swatches knit with alternating face knitting order; green paths marked with a square indicate swatches with entirely front face priority knitting order. Within each color, the bristle length is shown as shades from dark (shortest bristles, tuck distance 1) to light (longest bristles, tuck distance 3).

while the back slides forward. This "caterpillar" robot is soft and lightweight.

"Bristlebots" use springy bristles to turn vibration forces into net motion [41, 42]. Because our "bristle" structure can have knitin bias, we can produce fabrics which encourage linear motion to a greater or lesser extent (Figure 13). As noted in Section 6.3, this behavior shows some variation: the behavior of the front-facepriority bristlebots is somewhat inconsistent.

\subsection{Sensing and Soft Switches}

Knitting can incorporate areas of conductive yarns to support sensing [60]. For example, simple capacitive touch sensors [13] can be constructed from knitted-in conductive patches [2]. As with any soft sensor, care must be taken to interface with rigid circuit boards [49]; weft knitting simplifies this task because the traces themselves can be placed precisely within a knit, increasing the options for component placement.

Spacer fabrics lend themselves to several useful sensing configurations that can take advantage of their unique physical properties.

First: if both faces of the spacer fabric are knit with conductive yarns, and the filler yarn is non-conductive, the spacer structure can act as a force-sensitive capacitive touch sensor, Figure 14(a). We knit conductive yarn into areas of both faces of the spacer fabric. On face has edges knit with non-conductive yarn (shown in green) to prevent short circuits at the edges of the faces, where they curl and make contact. Using an off-the-shelf NXP Semiconductors MPR121 capacitive touch sensor chip attached to a microcontroller through an I2C interface, we were able to detect hover of a finger just above the surface of the face, touch of the surface, and most notably, movement of the two faces towards each other as pressure was applied to the top surface. This final capability is able to take advantage of the mechanical properties of the spacer fabric to create a type of soft compression sensor.
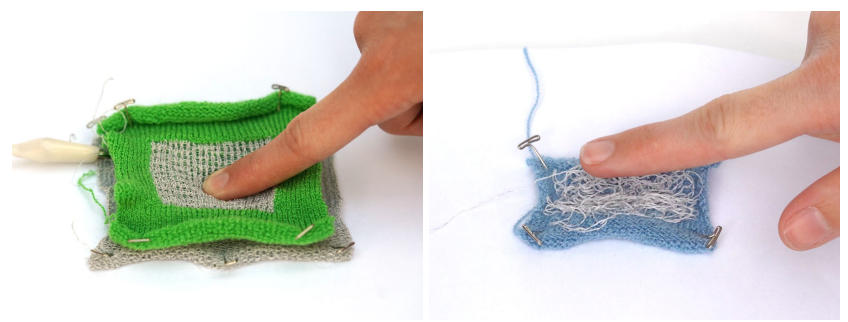

(a)

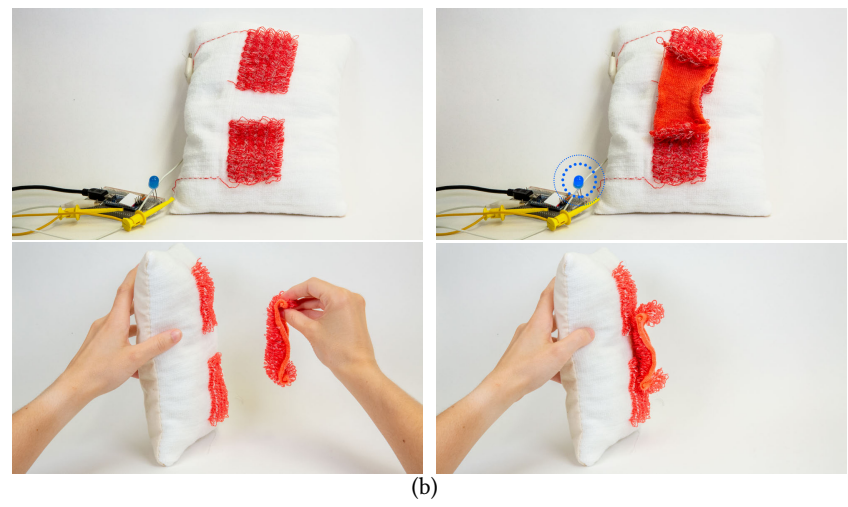

Figure 14: E-textiles swatches. (a) conductive yarn as the face yarn[s] for a pressure-sensitive capacitive sensor; conductive yarn as the filler for tactile conductive loops. (b) A "hook and loop"-style switch.

Second: 3D printed bristle patches have been shown to work as as a soft symmetric attachment similar to a hook and loop fastener [42]. Knit bristles can also achieve this effect if the filler yarn has high enough friction, such as a "felting" (not "superwash treated") wool yarn. By mixing a conductive yarn into the bristle 


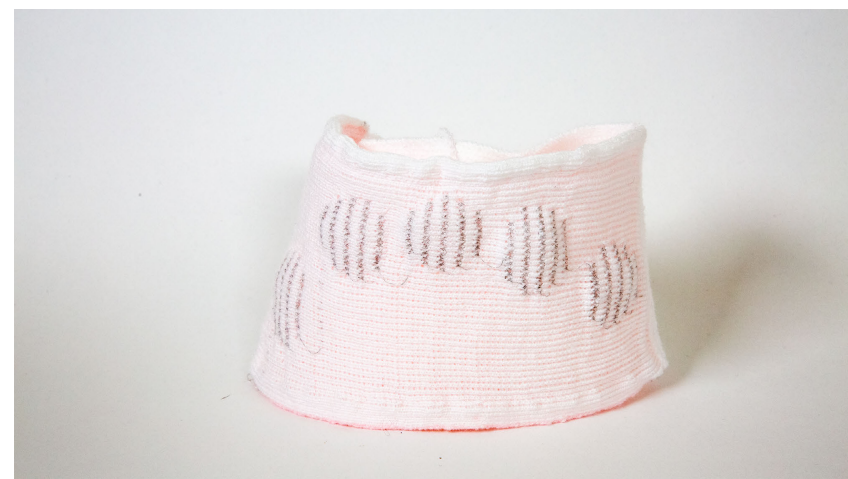

Figure 15: The soft control pad from Figure 1. Different thickness provide tactile cues to the location of capacitive button traces.

structure, we can make a soft switch, Figure 14(b): the bristles are knit with alternating rows of conductive and pure wool yarn as the filler, making the loops both conductive and self-sticky. The wool loops provide adhesion through high contact area friction, and the conductive loops carry electrical signal. The same swatch is very pleasant to the touch and can be used as a soft tactile "stroking sensor" inspired by [25].

Third: by combining capacitive sensing with tunable thickness, a button pad can be made which highlights interface areas with visible, tactile thickness differences. These differentially raised areas can be used to form custom collections of soft controls-in this case the geometry of a soft button pad has been formed directly within the fabric of an armband, Figure 7 (and Figure 1). For this application as a button pad, we used simple capacitive touch sensing on the top surface only. The conductive yarn was plated alongside the main face yarn in the contact areas.

All of our e-textile examples use Bekaert VN35X4/150POLY/350 (a stainless-steel and polyester yarn) as the conductive yarn.

\subsection{Integration into Knitted Objects}

Lastly, the flexibility of v-bed knitting as a process enables combining several knitting techniques within a knit object. Tendons can be incorporated within a face of knit fabric to transmit mechanical forces [2]; we combine tendon knitting with spacer fabric to produce a "pre-stuffed" knit tendon assembly suitable for soft gripping, Figure 16. The full assembly has a "palm" with multiple fingers, each with rounded fingertips. The assembly is knit sideways, in the direction parallel to the fingers, and uses a horizontal inlay for the tendons. After knitting, the structure is fully bound-off and stable and it ready to attach to a 3D-printed snap-together mount.

\section{LIMITATIONS AND FUTURE WORK}

While v-bed knitting as a fabrication process supports the broad range of spacer fabric characteristics and applications described in this work, designing the more complicated shapes remains a challenge. In particular the dual-layer structure of spacer fabric means that producing tubes, rather than sheets, presents significant planning challenges. Integrating spacer geometries as a "face type"

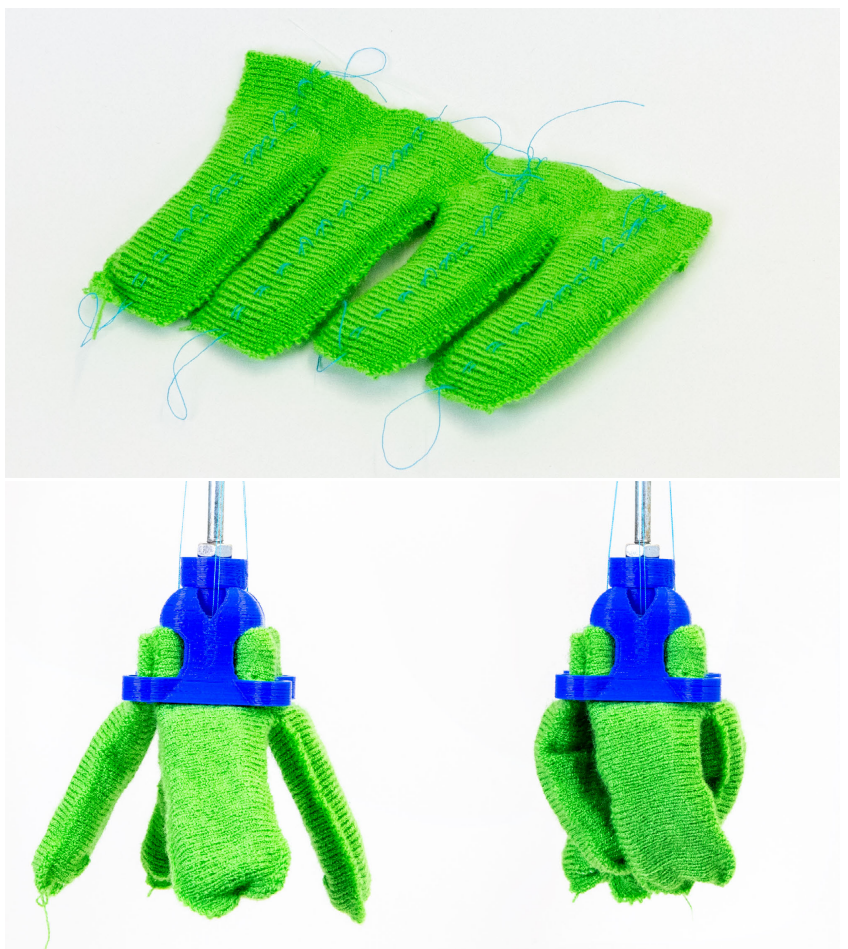

Figure 16: Fully-knit fingers for a soft gripper. Left: the assembly as-knit. Right: the gripper shown open and closed.

in system such as that proposed by Narayanan and Wu et al. [38] could ease this process. Additionally, using knit shaping techniques to produce spacer fabrics with large-scale curvature poses some mechanical challenges for typical machine-knit shaping techniques [2]: we found that transferring monofilament tucks was prone to errors, making increase/decrease shaping difficult, and the fabric's loftiness can cause short-row shaping to bunch and jam. We expect that future work in overall shaping with spacer fabrics will include calibration methods to articulate a spacer structure's knittability within a particular knitting machine's capabilities, as well as design tactics for separating the spacer and shaping sections of fabrics.

Lastly, our samples all used elastic to produce the draw-in that activates the spacer structure. Draw-in could also be produced with felting or heat-shrinking yarns. These could allow thickness to be set as a post-processing step, e.g. as a fabric that becomes thicker when triggered with heat or humidity (for use in, e.g., heat tailoring [57]).

\section{CONCLUSION}

We introduced weft-knit spacer fabrics as a programmable metamaterial and showed how a handful of knitting parameters can tune their mechanical characteristics for a variety of possible uses. We view such tunable fabric structures as foundational to the ongoing exploration of soft interfaces for robotics and wearables, as well as expanding the frontier of machine knitting as a uniquely powerful fabrication technology. 


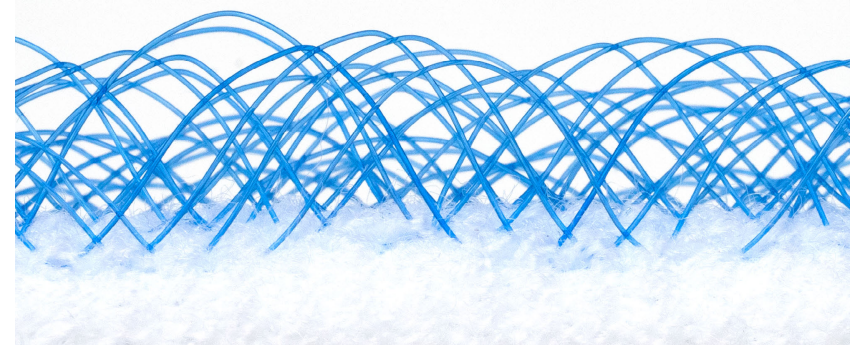

Figure 17: A close-up photograph of a monofilament bristle structure.

\section{ACKNOWLEDGMENTS}

The authors would like to thank David Renshaw for help with photography. This research was supported in part by National Science Foundation Grant IIS-1718651 and by NSF Engineering Research Center Planning Grant 1937031.

\section{REFERENCES}

[1] H.A. Abou-Taleb. 2014. Spacer Fabrics for Soft but Strong Knee Braces. Textile Asia 45 (Jan. 2014), 37-42.

[2] Lea Albaugh, Scott Hudson, and Lining Yao. 2019. Digital Fabrication of Soft Actuated Objects by Machine Knitting. In Proceedings of the 2019 CHI Conference on Human Factors in Computing Systems (CHI '19). Association for Computing Machinery, Glasgow, Scotland Uk, 1-13. https://doi.org/10.1145/3290605.3300414

[3] M. P. Bendsøe. 1989. Optimal Shape Design as a Material Distribution Problem. Structural Optimization 1, 4 (Dec. 1989), 193-202. https://doi.org/10.1007/ BF01650949

[4] Zhiyong Chen, Ronghui Wu, Shihui Guo, Xiangyang Liu, Hongbo Fu, Xiaogang Jin, and Minghong Liao. 2020. 3D Upper Body Reconstruction with Sparse Soft Sensors. Soft Robotics 0, 0 (July 2020), soro.2019.0187. https://doi.org/10.1089/ soro.2019.0187 arXiv:https://doi.org/10.1089/soro.2019.0187

[5] Inrak Choi, Nick Corson, Lizzie Peiros, Elliot W. Hawkes, Sean Keller, and Sean Follmer. 2018. A Soft, Controllable, High Force Density Linear Brake Utilizing Layer Jamming. IEEE Robotics and Automation Letters 3, 1 (Jan. 2018), 450-457. https://doi.org/10.1109/LRA.2017.2761938

[6] Mario de Araújo, Raul Fangueiro, André Catarino, and Hong Hu. 2001. Recent Developments in Weft-Knitting Science and Technology: The Way Ahead in the New Millennium. Revista Romana de Textile-Pielarie 1 (Jan. 2001), 61-66.

[7] Hassan M. El-Dessouky and Mohamed N. Saleh. 2018. 3D Woven Composites: From Weaving to Manufacturing. In Recent Developments in the Field of Carbon Fibers, Rita Khanna and Romina Cayumil (Eds.). IntechOpen, Rijeka, Chapter 4, 51-66. https://doi.org/10.5772/intechopen.74311

[8] M Glazzard. 2014. Re-Addressing the Role of Knitted Textile Design Knowledge: Auxetic Textiles from a Practice-Led, Designer-Maker Perspective. PhD Thesis. Nottingham Trent University.

[9] Antonio Gomes, Andrea Nesbitt, and Roel Vertegaal. 2013. MorePhone: An Actuated Shape Changing Flexible Smartphone. In CHI '13 Extended Abstracts on Human Factors in Computing Systems (CHI EA '13). Association for Computing Machinery, Paris, France, 2879-2880. https://doi.org/10.1145/2468356.2479558

[10] Jun Gong, Yu Wu, Lei Yan, Teddy Seyed, and Xing-Dong Yang. 2019. Tessutivo: Contextual Interactions on Interactive Fabrics with Inductive Sensing. In Proceedings of the 32nd Annual ACM Symposium on User Interface Software and Technology (UIST '19). Association for Computing Machinery, New York, NY, USA, 29-41. https://doi.org/10.1145/3332165.3347897

[11] Theodore Gray and Nina Paley. 2015. Quantitative Stretching.

[12] Anthony Grbic and George V. Eleftheriades. 2002. Experimental Verification of Backward-Wave Radiation from a Negative Refractive Index Metamaterial fournal of Applied Physics 92, 10 (Nov. 2002), 5930-5935. https://doi.org/10.1063/ 1.1513194

[13] Tobias Grosse-Puppendahl, Christian Holz, Gabe Cohn, Raphael Wimmer, Oskar Bechtold, Steve Hodges, Matthew S. Reynolds, and Joshua R. Smith. 2017. Finding Common Ground: A Survey of Capacitive Sensing in Human-Computer Interac tion. In Proceedings of the 2017 CHI Conference on Human Factors in Computing Systems (CHI '17). Association for Computing Machinery, New York, NY, USA, 3293-3315. https://doi.org/10.1145/3025453.3025808
[14] Claire Harvey, Emily Holtzman, Joy Ko, Brooks Hagan, Rundong Wu, Steve Marschner, and David Kessler. 2019. Weaving Objects: Spatial Design and Functionality of 3D-Woven Textiles. Leonardo 52, 4 (Aug. 2019), 381-388. https://doi.org/10.1162/leon_a_01780

[15] Megan Hofmann, Lea Albaugh, Ticha Sethapakadi, Jessica Hodgins, Scott E. Hudson, James McCann, and Jennifer Mankoff. 2019. KnitPicking Textures: Programming and Modifying Complex Knitted Textures for Machine and Hand Knitting. In Proceedings of the 32nd Annual ACM Symposium on User Interface Software and Technology (UIST '19). Association for Computing Machinery, New Orleans, LA, USA, 5-16. https://doi.org/10.1145/3332165.3347886

[16] D. Holland, E. J. Park, P. Polygerinos, G. J. Bennett, and C. J. Walsh. 2014. Actuators | Soft Robotics Toolkit. https://softroboticstoolkit.com/actuators.

[17] Cedric Honnet, Hannah Perner-Wilson, Marc Teyssier, Bruno Fruchard, Jürgen Steimle, Ana C Baptista, and Paul Strohmeier. 2020. PolySense: Augmenting Textiles with Electrical Functionality Using In-Situ Polymerization. In Proceedings of the 2020 CHI Conference on Human Factors in Computing Systems. ACM, Honolulu, HI, USA, 13. https://doi.org/10.1145/3313831.3376841

[18] Scott E. Hudson. 2014. Printing Teddy Bears: A Technique for 3D Printing of Soft Interactive Objects. In Proceedings of the 32nd Annual ACM Conference on Human Factors in Computing Systems - CHI '14. ACM Press, Toronto, Ontario, Canada, 459-468. https://doi.org/10.1145/2556288.2557338

[19] Alexandra Ion, Johannes Frohnhofen, Ludwig Wall, Robert Kovacs, Mirela Alistar, Jack Lindsay, Pedro Lopes, Hsiang-Ting Chen, and Patrick Baudisch. 2016. Metamaterial Mechanisms. In Proceedings of the 29th Annual Symposium on User Interface Software and Technology. ACM, Tokyo Japan, 529-539. https://doi.org/10.1145/2984511.2984540

[20] Alexandra Ion, Robert Kovacs, Oliver S. Schneider, Pedro Lopes, and Patrick Baudisch. 2018. Metamaterial Textures. In Proceedings of the 2018 CHI Conference on Human Factors in Computing Systems - CHI '18. ACM Press, Montreal QC, Canada, 1-12. https://doi.org/10.1145/3173574.3173910

[21] Alexandra Ion, David Lindlbauer, Philipp Herholz, Marc Alexa, and Patrick Baudisch. 2019. Understanding Metamaterial Mechanisms. In Proceedings of the 2019 CHI Conference on Human Factors in Computing Systems - CHI '19. ACM Press, Glasgow, Scotland Uk, 1-14. https://doi.org/10.1145/3290605.3300877

[22] Alexandra Ion, Ludwig Wall, Robert Kovacs, and Patrick Baudisch. 2017. Digital Mechanical Metamaterials. In Proceedings of the 2017 CHI Conference on Human Factors in Computing Systems. ACM, Denver Colorado USA, 977-988. https: //doi.org/10.1145/3025453.3025624

[23] Alexandre Kaspar, Liane Makatura, and Wojciech Matusik. 2019. Knitting Skeletons: A Computer-Aided Design Tool for Shaping and Patterning of Knitted Garments. In Proceedings of the 32nd Annual ACM Symposium on User Interface Software and Technology. ACM, New Orleans LA USA, 53-65. https: //doi.org/10.1145/3332165.3347879

[24] Jonathan P. King, Dominik Bauer, Cornelia Schlagenhauf, Kai-Hung Chang, Daniele Moro, Nancy Pollard, and Stelian Coros. 2018. Design. Fabrication, and Evaluation of Tendon-Driven Multi-Fingered Foam Hands. In 2018 IEEE-RAS 18th International Conference on Humanoid Robots (Humanoids). IEEE, Beijing, China, 1-9. https://doi.org/10.1109/HUMANOIDS.2018.8624997

[25] Kobakant. 2010. Knit Stroke Sensor. https://www.kobakant.at/DIY/?p=4317. (accessed 2020-02-27).

[26] Gierad Laput, Xiang 'Anthony' Chen, and Chris Harrison. 2015. 3D Printed Hair: Fused Deposition Modeling of Soft Strands, Fibers, and Bristles. In Proceedings of the 28th Annual ACM Symposium on User Interface Software \& Technology UIST '15. ACM Press, Daegu, Kyungpook, Republic of Korea, 593-597. https: //doi.org/10.1145/2807442.2807484

[27] Min Li, Shaokai Wang, Zuoguang Zhang, and Boming Wu. 2009. Effect of Structure on the Mechanical Behaviors of Three-Dimensional Spacer Fabric Composites. Applied Composite Materials 16, 1 (Feb. 2009), 1-14. https://doi.org/ 10.1007/s10443-008-9072-4

[28] Ruibo Liu, Qijia Shao, Siqi Wang, Christina Ru, Devin Balkcom, and Xia Zhou. 2019. Reconstructing Human Joint Motion with Computational Fabrics. Proceedings of the ACM on Interactive, Mobile, Wearable and Ubiquitous Technologies 3, 1 (March 2019), 19:1-19:26. https://doi.org/10.1145/3314406

[29] Yanping Liu and Hong Hu. 2010. Sound Absorption Behavior of Knitted Spacer Fabrics. Textile Research fournal 80, 18 (2010), 1949-1957. https://doi.org/10. 1177/0040517510373639

[30] Yanping Liu and Hong Hu. 2011. Compression Property and Air Permeability of Weft-knitted Spacer Fabrics. The fournal of The Textile Institute 102, 4 (2011), 366-372. https://doi.org/10.1080/00405001003771200

[31] James McCann. 2017. The "Knitout" (.k) File Format v0.5.3. https://textileslab.github.io/knitout/knitout.html. (accessed 2018-04-03).

[32] James McCann, Lea Albaugh, Vidya Narayanan, April Grow, Wojciech Matusik, Jennifer Mankoff, and Jessica Hodgins. 2016. A Compiler for 3D Machine Knitting. ACM Transactions on Graphics 35, 4 (July 2016), 49:1-49:11. https://doi.org/10. $1145 / 2897824.2925940$

[33] Gianluca Memoli, Letizia Chisari, Jonathan P. Eccles, Mihai Caleap, Bruce W. Drinkwater, and Sriram Subramanian. 2019. VARI-SOUND: A Varifocal Lens for Sound. In Proceedings of the 2019 CHI Conference on Human Factors in Computing 
Systems - CHI '19. ACM Press, Glasgow, Scotland Uk, 1-14. https://doi.org/10. $1145 / 3290605.3300713$

[34] Acme Mills. 2020. 3d Spacer Fabrics |Acme Mills. https://acmemills.com/brands/3d-spacer-fabrics.

[35] Satoshi Nakamaru, Ryosuke Nakayama, Ryuma Niiyama, and Yasuaki Kakehi. 2017. FoamSense: Design of Three Dimensional Soft Sensors with Porous Materials. In Proceedings of the 30th Annual ACM Symposium on User Interface Software and Technology (UIST '17). Association for Computing Machinery, Québec City, QC, Canada, 437-447. https://doi.org/10.1145/3126594.3126666

[36] Ryosuke Nakayama, Ryo Suzuki, Satoshi Nakamaru, Ryuma Niiyama, Yoshihiro Kawahara, and Yasuaki Kakehi. 2019. MorphIO: Entirely Soft Sensing and Actuation Modules for Programming Shape Changes through Tangible Interaction. In Proceedings of the 2019 on Designing Interactive Systems Conference. ACM, San Diego CA USA, 975-986. https://doi.org/10.1145/3322276.3322337

[37] Vidya Narayanan, Lea Albaugh, Jessica Hodgins, Stelian Coros, and James Mccann. 2018. Automatic Machine Knitting of 3D Meshes. ACM Transactions on Graphics 37, 3 (Aug. 2018), 1-15. https://doi.org/10.1145/3186265

[38] Vidya Narayanan, Kui Wu, Cem Yuksel, and James McCann. 2019. Visual Knitting Machine Programming. ACM Transactions on Graphics 38, 4 (July 2019), 63:163:13. https://doi.org/10.1145/3306346.3322995

[39] Alex Olwal, Jon Moeller, Greg Priest-Dorman, Thad Starner, and Ben Carroll 2018. I/O Braid: Scalable Touch-Sensitive Lighted Cords Using Spiraling, Repeating Sensing Textiles and Fiber Optics. In Proceedings of the 31st Annual ACM Symposium on User Interface Software and Technology (UIST '18). Association for Computing Machinery, Berlin, Germany, 485-497. https://doi.org/10.1145/ 3242587.3242638

[40] Alex Olwal, Thad Starner, and Gowa Mainini. 2020. E-Textile Microinteractions Augmenting Twist with Flick, Slide and Grasp Gestures for Soft Electronics. In Proceedings of the 2020 CHI Conference on Human Factors in Computing Systems (CHI '20). Association for Computing Machinery, Honolulu, HI, USA, 1-13. https: //doi.org/10.1145/3313831.3376236

[41] Windell H. Oskay and Lenore M. Edman. 2007. Bristlebot: A Tiny Directional Vibrobot. https://www.evilmadscientist.com/2007/bristlebot-a-tiny-directionalvibrobot/.

[42] Jifei Ou, Gershon Dublon, Chin-Yi Cheng, Felix Heibeck, Karl Willis, and Hiroshi Ishii. 2016. Cilllia: 3D Printed Micro-Pillar Structures for Surface Texture, Actuation and Sensing. In Proceedings of the 2016 CHI Conference on Human Factors in Computing Systems (CHI '16). Association for Computing Machinery, San Jose, California, USA, 5753-5764. https://doi.org/10.1145/2858036.2858257

[43] Jifei Ou, Daniel Oran, Don Derek Haddad, Joseph Paradiso, and Hiroshi Ishii. 2019 SensorKnit: Architecting Textile Sensors with Machine Knitting. 3D Printing and Additive Manufacturing 6, 1 (2019), 1-11. https://doi.org/10.1089/3dp.2018.0122

[44] Jifei Ou, Mélina Skouras, Nikolaos Vlavianos, Felix Heibeck, Chin-Yi Cheng, Jannik Peters, and Hiroshi Ishii. 2016. aeroMorph - Heat-Sealing Inflatable Shape-Change Materials for Interaction Design. In Proceedings of the 29th Annual Symposium on User Interface Software and Technology. ACM, Tokyo Japan, 121132. https://doi.org/10.1145/2984511.2984520

[45] Julian Panetta, Qingnan Zhou, Luigi Malomo, Nico Pietroni, Paolo Cignoni, and Denis Zorin. 2015. Elastic Textures for Additive Fabrication. ACM Transactions on Graphics 34, 4 (July 2015), 135:1-135:12. https://doi.org/10.1145/2766937

[46] Huaishu Peng, Jennifer Mankoff, Scott E. Hudson, and James McCann. 2015. A Layered Fabric 3D Printer for Soft Interactive Objects. In Proceedings of the 33rd Annual ACM Conference on Human Factors in Computing Systems (CHI '15) Association for Computing Machinery, Seoul, Republic of Korea, 1789-1798. https://doi.org/10.1145/2702123.2702327

[47] Andreas Pointner, Thomas Preindl, Sara Mlakar, Roland Aigner, and Michael Haller. 2020. Knitted RESi: A Highly Flexible, Force-Sensitive Knitted Textile Based on Resistive Yarns. In Special Interest Group on Computer Graphics and Interactive Techniques Conference Emerging Technologies. ACM, Virtual Event USA, 1-2. https://doi.org/10.1145/3388534.3407292

[48] Mariana Popescu, Matthias Rippmann, Tom Van Mele, and Philippe Block 2018. Automated Generation of Knit Patterns for Non-Developable Surfaces. In Humanizing Digital Reality, Klaas De Rycke, Christoph Gengnagel, Olivier Baverel, Jane Burry, Caitlin Mueller, Minh Man Nguyen, Philippe Rahm, and Mette Ramsgaard Thomsen (Eds.). Springer Singapore, Singapore, 271-284. https://doi.org/10.1007/978-981-10-6611-5_24

[49] Ivan Poupyrev, Nan-Wei Gong, Shiho Fukuhara, Mustafa Emre Karagozler, Carsten Schwesig, and Karen E. Robinson. 2016. Project Jacquard: Interac tive Digital Textiles at Scale. In Proceedings of the $2016 \mathrm{CHI}$ Conference on Human Factors in Computing Systems. ACM, San Jose California USA, 4216-4227. https://doi.org/10.1145/2858036.2858176

[50] Thomas Preindl, Cedric Honnet, Andreas Pointner, Roland Aigner, Joseph A Paradiso, and Michael Haller. 2020. Sonoflex: Embroidered Speakers without Permanent Magnets. In UIST'20: 33rdth ACM User Interface Software and Technology Symposium. ACM, Minneapolis, Minnesota, USA, 8.

[51] Ahmad Rafsanjani, Yuerou Zhang, Bangyuan Liu, Shmuel M. Rubinstein, and Katia Bertoldi. 2018. Kirigami Skins Make a Simple Soft Actuator Crawl. Science Robotics 3, 15 (Feb. 2018), eaar7555. https://doi.org/10.1126/scirobotics.aar7555
[52] Michael L. Rivera and Scott E. Hudson. 2019. Desktop Electrospinning: A Single Extruder 3D Printer for Producing Rigid Plastic and Electrospun Textiles. In Proceedings of the 2019 CHI Conference on Human Factors in Computing Systems - CHI '19. ACM Press, Glasgow, Scotland Uk, 1-12. https://doi.org/10.1145/ 3290605.3300434

[53] Andreas Roye and Thomas Gries. 2007. 3-D Textiles for Advanced Cement Based Matrix Reinforcement. Fournal of Industrial Textiles 37, 2 (2007), 163-173. https://doi.org/10.1177/1528083707078136

[54] Robert T. Spillane and Mike S. Kowalski. 1995. Warp Knitted Textile Spacer Fabric, Method of Producing Same, and Products Produced Therefrom. Patent No. US5385036A, Filed 1993-05-24, Issued 1995-01-31.

[55] Ruojia Sun, Ryosuke Onose, Margaret Dunne, Andrea Ling, Amanda Denham, and Hsin-Liu (Cindy) Kao. 2020. Weaving a Second Skin: Exploring Opportunities for Crafting On-Skin Interfaces Through Weaving. In Proceedings of the 2020 ACM Designing Interactive Systems Conference (DIS '20). Association for Computing Machinery, Eindhoven, Netherlands, 365-377. https://doi.org/10.1145/3357236. 3395548

[56] James Utama Surjadi, Libo Gao, Huifeng Du, Xiang Li, Xiang Xiong, Nicholas Xuanlai Fang, and Yang Lu. 2019. Mechanical Metamaterials and Their Engineering Applications. Advanced Engineering Materials 21, 3 (2019), 1800864. https://doi.org/10.1002/adem.201800864

[57] Lavender Tessmer, Carmel Dunlap, Bjorn Sparrman, Schendy Kernizan, Jared Laucks, and Skylar Tibbits. 2019. Active Textile Tailoring. In ACM SIGGRAPH 2019 Emerging Technologies (SIGGRAPH '19). Association for Computing Machinery, New York, NY, USA, 2. https://doi.org/10.1145/3305367.3327995

[58] Jenny Underwood. 2009. The Design of 3D Shape Knitted Preforms. Ph.D. Dissertation. RMIT University.

[59] Guanyun Wang, Tingyu Cheng, Youngwook Do, Humphrey Yang, Ye Tao, Jianzhe Gu, Byoungkwon An, and Lining Yao. 2018. Printed Paper Actuator: A Low-Cost Reversible Actuation and Sensing Method for Shape Changing Interfaces. In Proceedings of the 2018 CHI Conference on Human Factors in Computing Systems CHI '18. ACM Press, Montreal QC, Canada, 1-12. https://doi.org/10.1145/3173574. 3174143

[60] Jinfeng Wang, Hairu Long, Saeid Soltanian, Peyman Servati, and Frank Ko. 2014. Electromechanical Properties of Knitted Wearable Sensors: Part 1 - Theory. Textile Research fournal 84, 1 (Jan. 2014), 3-15. https://doi.org/10.1177/ 0040517513487789

[61] Lijing Wang. 2008. The Use of Spacer Fabrics for Absorbent Medical Applications. fournal of Fiber Bioengineering and Informatics 1, 4 (2008), 321-329. https: //doi.org/10.3993/jfbi03200910

[62] Ting Xu and Henri J. Lezec. 2014. Visible-Frequency Asymmetric Transmission Devices Incorporating a Hyperbolic Metamaterial. Nature Communications 5, 1 (June 2014), 4141. https://doi.org/10.1038/ncomms5141

[63] MIAO Xu-hong and Ge Ming-Oiao. 2008. The Compression Behaviour of Warp Knitted Spacer Fabric. Fibres \& Textiles in Eastern Europe 16, 1 (2008), 90-92.

[64] Lining Yao, Ryuma Niiyama, Jifei Ou, Sean Follmer, Clark Della Silva, and Hiroshi Ishii. 2013. PneUI: Pneumatically Actuated Soft Composite Materials for Shape Changing Interfaces. In Proceedings of the 26th Annual ACM Symposium on User Interface Software and Technology - UIST '13. ACM Press, St. Andrews, Scotland, United Kingdom, 13-22. https://doi.org/10.1145/2501988.2502037

[65] Hechuan Zhang, Zhiyong Chen, Shihui Guo, Juncong Lin, Yating Shi, Xiangyang Liu, and Yong Ma. 2020. Sensock: 3D Foot Reconstruction with Flexible Sensors. In Proceedings of the 2020 CHI Conference on Human Factors in Computing Systems (CHI '20). Association for Computing Machinery, Honolulu, HI, USA, 1-13. https: //doi.org/10.1145/3313831.3376387

[66] Mengjia Zhu, Amirhossein H. Memar, Aakar Gupta, Majed Samad, Priyanshu Agarwal, Yon Visell, Sean J. Keller, and Nicholas Colonnese. 2020. PneuSleeve: In-Fabric Multimodal Actuation and Sensing in a Soft, Compact, and Expressive Haptic Sleeve. In Proceedings of the 2020 CHI Conference on Human Factors in Computing Systems (CHI '20). Association for Computing Machinery, Honolulu, HI, USA, 1-12. https://doi.org/10.1145/3313831.3376333

\section{A IMPLEMENTATION NOTES}

We offer additional knitting details here to encourage further exploration with these structures.

These tips are based on our experience with our Shima Seiki SWG091N2 (15 gauge) v-bed knitting machine. 


\section{A.1 Filler Yarn}

The filament must be stiff enough to provide loft but supple enough to run through the machine's tensioning apparatus. We used KastKing $6 \mathrm{lb}$ test strength monofilament $(0.22 \mathrm{~mm} \varnothing)$. Because monofilament is typically wound on rolls instead of cones, and even a small amount of twist in the monofilament caused tangles, we found we needed to rig a passive roll feeder and run the machine at approximately $1 / 5$ of our normal carriage speed during tucking rows for smooth feeding. Additionally, we found that using a very shallow stitch size for the "tuck" operations which incorporate the monofilament helped to prevent the monofilament from poking through the faces and disrupting subsequent knit loops. In the units used by our knitting machine, we used "stitch size" 25 and "leading set" 25 for these tucks, while our face knit loops were formed at stitch size 40 (leading set 25).

\section{A.2 Elastic}

We used a hair-thin latex elastic that could provide face draw-in while allowing our choice of structural face yarns. This elastic is very fragile and must be knit at consistent tension to provide consistent draw-in. We used our machine's elastic yarn tensioning system, which actively advances the yarn by a consistent amount per stitch formed. However, one major machine constraint is that some knitting machines may not have multiple elastic yarn carriers, as ours does not, so we could not knit two entirely separate faces in a time-efficient way (i.e., without cutting the yarn after every face row). For examples that required fully-separated faces, we used a sewing seam ripper to sever just the elastic in order to separate the faces after knitting.

\section{A.3 Face Yarns}

We saw the best results with relatively fine yarns used in the facesa less-dense face allows "room to collapse," encouraging draw-in for greater spacer thickness. While our structures were knit at halfgauge (simulated $7 \mathrm{~g}$ ), we primarily knit with the Tamm "Petit" yarn that we typically use for full-gauge $(15 \mathrm{~g})$.

\section{A.4 Yarn Tangling}

With at least four carriers (two face yarns, one elastic, and one filler yarn) in play at the same time, some amount of yarn carrier tangling is difficult to avoid. To prevent accidentally "stitching" the faces together, the tuck pattern incorporates an extra tuck on one face to keep the filler yarn in a consistent direction from the elastic yarn.

\section{A.5 Production Time}

Production time primarily increases with the additional carriage passes required for the tuck rows-as summarized in Figure 8, these additional passes range from one filament row per four face rows to five per single face row-plus some small constant time ( $30 \mathrm{sec}$ ) to bring additional yarn feeders into play. Therefore, when combined with the slower speeds during monofilament rows as described above, our spacer fabric knits took between 2 and 25 times as long as comparable knits without filler rows, depending on filler row density and yarn choice. The fabric portion of Figure 16 was knit in approximately an hour. 